\title{
Theoretical evidence for unexpected O-rich phases at corners of MgO surfaces
}

\author{
Saswata Bhattacharya, ${ }^{1, *}$ Daniel Berger, ${ }^{2}$ Karsten Reuter, ${ }^{2}$ Luca M. Ghiringhelli, ${ }^{3}$ and Sergey V. Levchenko ${ }^{3,4, *}$ \\ ${ }^{1}$ Department of Physics, Indian Institute of Technology Delhi, New Delhi 110016, India \\ ${ }^{2}$ Chair for Theoretical Chemistry and Catalysis Research Center, Technical University Münich, \\ Lichtenbergstrasse 4, D-85747 Garching, Germany \\ ${ }^{3}$ Fritz-Haber-Institut der Max-Planck-Gesellschaft, Faradayweg 4-6, D-14195 Berlin, Germany \\ ${ }^{4}$ NUST MISIS, Moscow, Russia
}

(Received 18 June 2017; published 13 December 2017)

\begin{abstract}
Realistic oxide materials are often semiconductors, in particular at elevated temperatures, and their surfaces contain undercoordinated atoms at structural defects such as steps and corners. Using hybrid density-functional theory and ab initio atomistic thermodynamics, we investigate the interplay of bond-making, bond-breaking, and charge-carrier trapping at the corner defects at the (100) surface of a p-doped $\mathrm{MgO}$ in thermodynamic equilibrium with an $\mathrm{O}_{2}$ atmosphere. We show that by manipulating the coordination of surface atoms, one can drastically change and even reverse the order of stability of reduced versus oxidized surface sites.
\end{abstract}

DOI: 10.1103/PhysRevMaterials.1.071601

In surface catalysis, point defects often play the role of active sites. It is widely accepted that $\mathrm{O}$ vacancies are important active sites at metal-oxide surfaces $[1,2]$. This implies that the concentration of the vacancies at reaction conditions is sufficiently high (above a few parts per million). Typically, defect properties are studied under ultrahigh-vacuum conditions, when the concentration is higher due to low oxygen partial pressure $p_{\mathrm{O}_{2}}$. It is therefore important to explore how many vacancies remain stable under the O-rich conditions of technological catalysis.

Impurities and intrinsic defects often convert materials that are ideally band-gap insulators (e.g., $\mathrm{TiO}_{2}, \mathrm{ZnO}, \mathrm{MgO}$ ) into semiconductors. In addition to the effect on the electronic properties of a material, this charge-carrier doping (either accidental or intentional) can also influence the material's surface chemistry [3]. Oxygen vacancies provide electronic states deep in the band gap that can accommodate the holes. As a result, the free energy of the system is lowered, and the vacancies get locally charged. Even at low doping levels $\left(<10^{17} \mathrm{~cm}^{-3}\right)$, the concentration of such charged $\mathrm{O}$ vacancies at a $\mathrm{MgO}(100)$ terrace exceeds 0.01 at. $\%$ at $T=400 \mathrm{~K}$ and $p_{\mathrm{O}_{2}}=1$ atm [3].

Realistic oxide surfaces, however, are rarely atomically flat. They contain structural defects such as steps and corners, where atoms have a coordination different from the flat surface (terrace) [4]. These defect sites can play an important role in catalytic reactions, even at low concentrations, since they are usually much more reactive than the terrace sites. Although the concentration and stability of such sites at realistic conditions remain debatable, there is combined experimental/theoretical evidence that they indeed play an important role in catalysis [5-9]. In general, it seems reasonable to expect that the formation energy of an oxygen vacancy at a step or corner is lower than at the terrace, since the number of bonds to be broken is smaller. Indeed, the formation energy of a neutral $\mathrm{O}$ vacancy at steps and corners at the $\mathrm{MgO}(100)$ surface is lower than at the terrace $[10,11]$. However, it is not clear a priori

\footnotetext{
*saswata@physics.iitd.ac.in and levchenko@fhi-berlin.mpg.de
}

how the presence of the structural defects will influence the charge-carrier trapping energy. Moreover, we have recently shown that small $\mathrm{MgO}$ clusters in thermodynamic equilibrium with an $\mathrm{O}_{2}$ atmosphere contain an excess rather than deficiency of oxygen compared to the stoichiometric oxide, even at high temperatures, exactly due to the presence of the undercoordinated sites [12]. Thus, the type of the most abundant defect at the steps, and in particular corners of a doped oxide at realistic conditions, should be determined by a nontrivial interplay between the site coordination and the charge-carrier trapping.

In this paper, we address the above problem. Our material of choice is $\mathrm{MgO}$ - an important catalytic material and a widely studied prototypical system. We focus on the corners at the $p$-doped [13] MgO (100) surface, since the differences with the terrace are expected to be more pronounced in this case. The step edges will be addressed elsewhere. $\mathrm{MgO}$ surfaces with a high concentration of corner sites can be prepared by annealing $\mathrm{MgO}$ (111) surfaces or by producing nanocrystalline $\mathrm{MgO}$. We calculate relative concentrations of corner defects of different types (vide infra the O-vacancy as well as $\mathrm{O}_{1} / \mathrm{O}_{2}$-ad-species) using density-functional theory (DFT) and $a b$ initio atomistic thermodynamics [14]. The $\mathrm{O}$ and $\mathrm{O}_{2}$ moieties adsorbed at corners at $\mathrm{MgO}$ (100) surface have been extensively studied previously $[4,15]$. However, their stability at realistic temperature, pressure, and doping conditions has so far remained unclear. In our work, the doping is considered as a means of fixing the chemical potential of the electrons ( $\mu_{e}$; see below). We note that charge carriers can also be produced by photoexcitation, so that our findings may be relevant for photocatalytic reactions as well.

Corner defects are modeled by embedded clusters (see Fig. 1). Corners separated from the underlying surface by one, two, or an infinite number of layers are considered. We use a QM/MM-type embedding [16], where an atomic cluster treated at the $a b$ initio level [the quantum-mechanical (QM) part] is embedded into a region treated at the level of empirical interatomic potentials [the molecular-mechanics (MM) part]. Cations (Mg sites), which are next neighbors to sites treated at the $a b$ initio level, are described by norm-conversing nonlocal pseudopotentials [17]. The ab initio calculations are performed 




FIG. 1. (a) Periodic model of the $\mathrm{Mg}_{80} \mathrm{O}_{80}$ corner. (b) $\mathrm{Mg}_{80} \mathrm{O}_{80}$, $\mathrm{Mg}_{25} \mathrm{O}_{25}$, and $\mathrm{Mg}_{32} \mathrm{O}_{32}$ clusters, and (c) the corresponding embedded cluster models for $\mathrm{Mg}_{25} \mathrm{O}_{25}$ and $\mathrm{Mg}_{32} \mathrm{O}_{32}$ (the $\mathrm{Mg}_{80} \mathrm{O}_{80}$ embedded model is not shown). The red (blue) spheres represent $\mathrm{O}(\mathrm{Mg})$ atoms in the QM region; white spheres are point charges $\left(+2\right.$ for $\mathrm{Mg}^{2+}$ and -2 for $\mathrm{O}^{2-}$ ) fixed to the perfect lattice positions; gray (yellow) spheres are the polarizable $-2(+2)$ charges (see the text).

with the all-electron FHI-aims program package [18], where the $\mathrm{QM} / \mathrm{MM}$ embedding infrastructure has been recently implemented [19,20] [see the Supplemental information (SI) [21]].

The QM region is treated using DFT. The full atomic relaxation is done using the generalized gradient exchangecorrelation (xc) functional PBE [22,23]. Total energy calculations are performed at these geometries with the hybrid $\mathrm{xc}$ functional HSE06 [24]. First-principles dispersion correction is included via the Tkatchenko-Scheffler scheme [25] for all clusters. The high-accuracy basis sets and numerical grids are defined by the tight settings [18] that have been used throughout our calculations.

To identify the relevant defect structures for the QM region, we have studied the relative stability of a set of parallelepipedal $\left.\left[\mathrm{Mg}_{N} \mathrm{O}_{(x+N)}\right)\right]^{q}$ clusters of various sizes $[N=18,24,32,108]$ with a defect at one of the corners $[x=-1$ (O-vacancy), 0 (pristine), 1 ( $\mathrm{O}_{1}$-ad-species $), 2\left(\mathrm{O}_{2}\right.$-ad-species $\left.)\right]$. The charge state $q$ is varied from -2 to $+2[q=-2,-1,0,1,2]$. By using our cascade genetic algorithm (cGA) [12,26], we found that for all defects, global minimum structures have the defect located at the O-terminated corner and not at the edges or faces of the parallelepipedal structures (Fig. 3, lower panel). The greater stability of the oxygen ad-species at the $\mathrm{O}$ - versus $\mathrm{Mg}$-terminated corner is explained by the stronger basicity of the undercoordinated $\mathrm{O}$ atom that can donate electrons to the additional electrophilic $\mathrm{O}$ species. For an accurate description of defect charging, the embedding scheme should closely reproduce the electronic structure of the corresponding extended system, in particular the position of the defect levels with respect to (w.r.t.) the bulk Fermi level. We check this by comparing the electronic structure of the embedded cluster model of a pristine O-terminated corner with a periodic slab model (see SI) [21].
We analyze the thermodynamic stability of the defected clusters w.r.t. the pristine clusters by means of $a b$ initio atomistic thermodynamics. We consider a reservoir of gasphase $\mathrm{O}_{2}$ molecules, characterized by the chemical potential $\mu_{\mathrm{O}_{2}}$. For clusters $\left[\mathrm{Mg}_{N} \mathrm{O}_{(N+x)}\right]^{q}$ with charge $q$, the formation Gibbs free energy $\left[\Delta G^{q}\left(T, p_{\mathrm{O}_{2}}\right)\right]$ w.r.t. the host (neutral stoichiometric cluster) is

$$
\Delta G^{q}\left(T, p_{\mathrm{O}_{2}}\right)=\Delta E_{\mathrm{f}}-x \Delta \mu_{\mathrm{O}}\left(T, p_{\mathrm{O}_{2}}\right)+q \mu_{\mathrm{e}}
$$

where $\Delta E_{\mathrm{f}}=E_{\text {defect }}^{q}-E_{\text {host }}^{q=0}-\frac{x}{2} E_{\mathrm{O}_{2}}$ is the DFT defect formation energy, $\Delta \mu_{\mathrm{O}}=\frac{1}{2}\left(\mu_{\mathrm{O}_{2}}-E_{\mathrm{O}_{2}}\right)$, and $E_{\mathrm{O}_{2}}, E_{\text {defect }}^{q}, E_{\text {host }}^{q=0}$ are the total energies of the oxygen molecule, the cluster with a defect, and the cluster without a defect, respectively. Vibrational contributions to the free energies of formation are found to be around $0.1 \mathrm{meV}$ for the systems studied here, and they are therefore neglected.

We find that at realistic temperatures and pressures (e.g., $\left.T=300 \mathrm{~K}, p_{\mathrm{O}_{2}} \sim 1 \mathrm{~atm}\right), \mathrm{O}_{1}$ - or $\mathrm{O}_{2}$-ad-species at the oxygen corners of pristine $\left[(\mathrm{MgO})_{N}\right]^{q}$ clusters are favored for all studied charge states over a wide range of electronic chemical potential $\mu_{e}$ (phase diagrams are given in the SI [21]). In fact, the $\mathrm{O}_{1} / \mathrm{O}_{2}$-ad-species are stable even at some values of $\mu_{e}$, for which O-vacancies are preferred at flat (100) surfaces of p-doped $\mathrm{MgO}$.

Following the findings for the isolated parallelepipedal clusters, we have created several embedded cluster models, taking as a starting point for the QM region $\mathrm{Mg}_{32} \mathrm{O}_{32+x}$ [Fig. 1(b), bottom right cubic structure], $\mathrm{Mg}_{25} \mathrm{O}_{25+x}$ [Fig. 1(b), bottom left step structure], and $\mathrm{Mg}_{20} \mathrm{O}_{21+x}$ (not shown). Here, $x=0$ represents the pristine corners, while $x=-1$, 1 , and 2 are, respectively, $\mathrm{O}$-vacancy, $\mathrm{O}_{1}$-ad-species, and $\mathrm{O}_{2}$-ad-species. These clusters are embedded in the $\mathrm{MM}$ regions as explained above [see Fig. 1(c)]. The initial geometries for the embedded clusters are created by cutting out the defected corner (for $x=-1,1$, and 2) and its nearest neighbors from the isolated parallelepipedal clusters and replacing it with the corresponding part at the corner of interest of the otherwise perfect embedded cluster. The number of relaxed atoms in the QM region was expanded until the convergence of the formation energies of the defects is achieved. Similarly, convergence of the electrostatic potential w.r.t. the number of point charges is also thoroughly tested (see the SI) [21].

The thermodynamic stability of the corner defects at an extended (100) surface of $p$-doped $\mathrm{MgO}$ was then studied using the embedded cluster models and Eq. (1). $E_{\text {defect }}^{q}$ now refers to the embedded cluster with $x$ additional (removed when $x=-1$ ) oxygen atoms and a charge $q$, while the host is the stoichiometric neutral embedded cluster. Here we show results for the defects at the corners formed by O-terminated multilayer (three or more layers) step edges. The results for the corners formed by two-layer and monolayer step edges are similar (see the SI [21]). The calculated phase diagram is shown in Fig. 2. Clearly, $\mathrm{O}_{1} / \mathrm{O}_{2}$-ad-species are stabilized also at the corners at the extended surface. Note that the extra stability of $\mathrm{O}_{1} / \mathrm{O}_{2}$-ad-species over $\mathrm{O}$-vacancies is preserved over a wide range of $\mu_{e}$ (see the SI [21]).

We also calculate the concentration of the defects as a function of the thermodynamic parameters. In the limit of a small concentration of the corners, when the interaction 


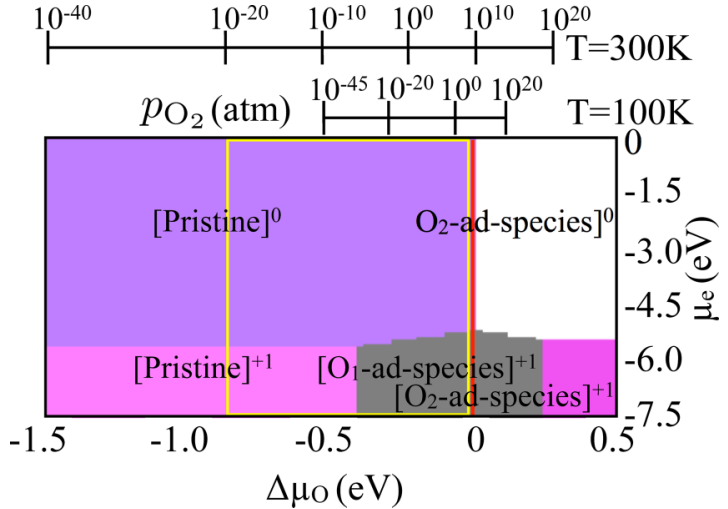

FIG. 2. Phase diagram for defects at corners of $\mathrm{MgO}$ (100) in an oxygen atmosphere, calculated using embedded $\left[\mathrm{Mg}_{32} \mathrm{O}_{32+x}\right]^{q}$ clusters, with $x=-1$ (vacancy), 0 (pristine), 1 ( $\mathrm{O}_{1}$-ad-species), and $2\left(\mathrm{O}_{2}\right.$-ad species $)$, and $q=-2,1,0,1$, and 2 . The energy zero on the $\mu_{e}$ axis corresponds to the vacuum level. [This $\mu_{e}$ includes the shift associated with the band bending due to the formation of $\mathrm{O}$ vacancies at terraces (see the text)]. The yellow rectangle represents the region that is relevant for catalytic applications, while the red vertical line represents the O-rich limit.

between them can be neglected, the defects will obey the Fermi-Dirac statistics (as identical particles that cannot occupy the same site more than once). As has been shown previously [3], formation of $\mathrm{O}$ vacancies at the (001) surface of $p$-doped $\mathrm{MgO}$ at realistic $\left(T, p_{\mathrm{O}_{2}}\right)$ conditions and doping concentrations $N_{\mathrm{D}}$ results in the formation of a space-charge layer under the surface and concomitant band bending. For a small concentration of the corner sites, the contribution of defects at these sites to the band bending can be neglected. In this case, the effect of the band bending on the formation energies of defects at corners is simply taken into account by shifting $\mu_{e}$ in Eq. (1) upwards by the value of the band bending (since the band bending shifts the defect electronic levels down with respect to the bulk Fermi level), which is a function of $T, p_{\mathrm{O}_{2}}$, and $N_{\mathrm{D}}[3]$.

If $N$ is the total number of corners and $\Delta G_{n}$ is the formation Gibbs free energy relative to the pristine neutral corner of a given type- $n$ defect, then the number of corners with type- $n$ defect, $N_{n}$, is

$$
N_{n}=\left(N-\Sigma_{m \neq n} N_{m}\right) \frac{1}{\exp \left(\beta \Delta G_{n}\right)+1},
$$

where $\beta=1 / k_{\mathrm{B}} T$, and $N_{m}$ is any other considered defect type. A similar equation is written for each considered defect type. The solution of the set of coupled equations is

$$
\frac{N_{n}}{N}=\frac{\exp \left(-\beta \Delta G_{n}\right)}{1+\Sigma_{m} \exp \left(-\beta \Delta G_{m}\right)} .
$$

Figure 3 shows the concentration of different kinds of defects at varying temperature and constant pressure. At $\mu_{e}=-7.5 \mathrm{eV}$ and $p_{\mathrm{O}_{2}}=1 \mathrm{~atm}, \mathrm{O}_{1}$-ad-species with a trapped hole $\left(\left[\mathrm{O}_{1} \text {-ad-species }\right]^{+1}\right.$ in Fig. 3) at the corners of $\mathrm{MgO}$ surface are the most abundant species at all temperatures. Only at temperatures around $900 \mathrm{~K}$ and above does another defect start to compete with it, namely the pristine corner with a trapped hole ([Pristine $]^{+1}$ in Fig. 3). At the high temperature,

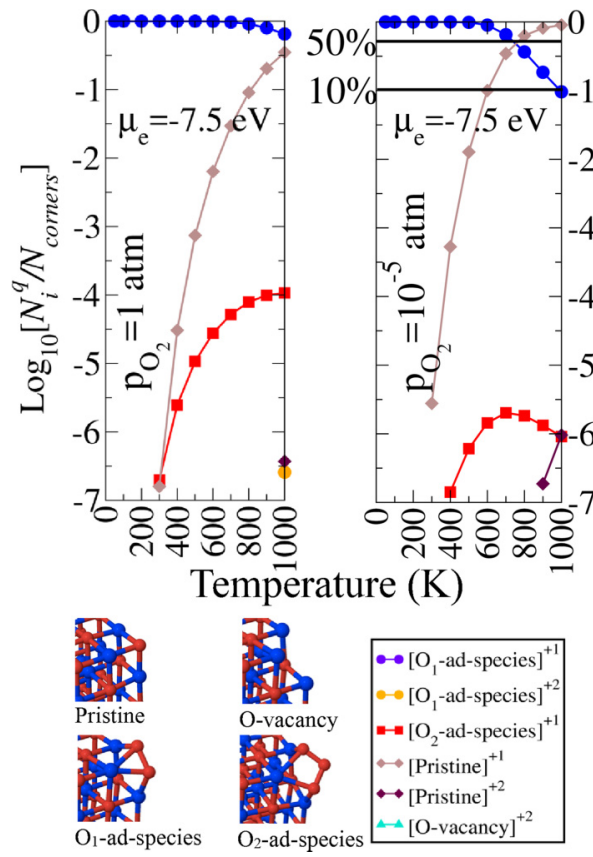

FIG. 3. Concentration of defects at corners at $p_{\mathrm{O}_{2}}=1 \mathrm{~atm}$ (left) and $p_{\mathrm{O}_{2}}=10^{-5}$ atm (right) for $p$-doped $\mathrm{MgO}\left(\mu_{e}=-7.5 \mathrm{eV}\right)$. The calculated concentrations include the effects of band bending for a dopant concentration $\mathrm{N}_{\mathrm{D}}=10^{16} \mathrm{~cm}^{-3}$. There is a negligible change in the defect concentrations when the dopant concentration is increased to $10^{22} \mathrm{~cm}^{-3}$

about $0.01 \%$ of all O-terminated corners should have an $\mathrm{O}_{2}$ molecule adsorbed on them, again with a trapped single hole $\left(\left[\mathrm{O}_{2} \text {-ad-species }\right]^{+1}\right.$ in Fig. 3). Interestingly, surface $\mathrm{O}_{3}^{-}$ species have been previously proposed to explain transmission electron diffraction measurements of reconstructed $\mathrm{MgO}$ (111) surfaces, which are expected to expose a high coverage of corner sites [27]. In fact, metal-superoxo and metal-ozonide complexes and their interconversion play an important role in organometallic chemistry and biochemistry, where they are well known as $\eta$ side groups. In our case, however, the fine-tuning of the chemical reactivity of metal centers is achieved by controlling the concentration of charge carriers and coordination at the inorganic surface sites, instead of organic ligands.

The estimated error of $\pm 0.2 \mathrm{eV}$ in the position of the defect levels w.r.t. the electronic chemical potential $\left(\mu_{e}\right)$ has no qualitative effect on the calculated relative concentrations (see SI [21]). At lower $\mathrm{O}_{2}$ pressures, the pristine corner with a trapped hole ([Pristine $]^{+1}$ in Fig. 3) starts to compete with $\left[\mathrm{O}_{1} \text {-ad-species }\right]^{+1}$ at lower temperatures, but these two defects remain dominant in the considered temperature range. Another interesting result is that the concentration of oxygen vacancies with two trapped holes ([O-vacancy $]^{+2}$ ) at corners is found to be negligible at realistic conditions, which is in drastic contrast to the terrace sites.

We have checked the stability of an $\mathrm{O}$ atom adsorbed at the (001) terrace of $p$-doped $\mathrm{MgO}$ in charge states $0,+1$, and +2 . According to our HSE06 results, the adsorbed $\mathrm{O}$ atom is at least $1.8 \mathrm{eV}$ less stable (depending on the charge state) than the doubly charged $\mathrm{O}$ vacancy in the dilute limit (and by $1.3 \mathrm{eV}$ for 


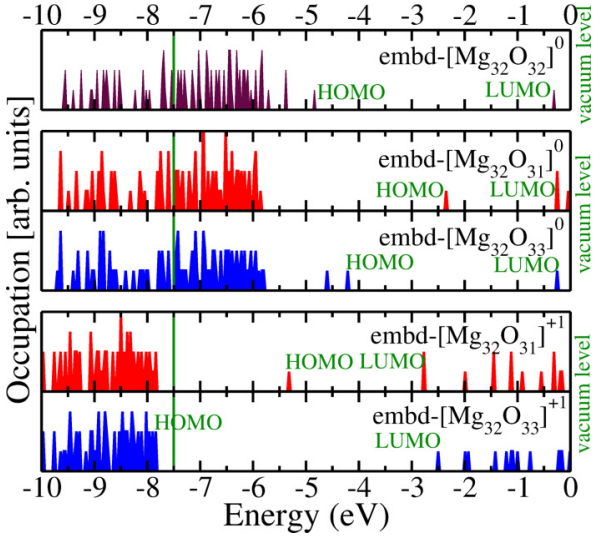

FIG. 4. Kohn-Sham spectrum of a neutral embedded $\mathrm{Mg}_{32} \mathrm{O}_{32+x}$ cluster, with $x=-1$ (vacancy) and $x=1$ (O ad-species).

one defect per $2 \times 2$ surface unit cell) even under most O-rich conditions $\left(\mu_{\mathrm{O}}=0\right)$. Thus, although at corners $\mathrm{O}$ ad-atoms are abundant defects, at the terraces their concentration is very small, and their effect on the band bending can be neglected.

To understand the extra stability of $\mathrm{O}_{1} / \mathrm{O}_{2}$-ad-species over $\mathrm{O}$ vacancies at the corners of the $\mathrm{MgO}$ surface, we analyze the density of states of embedded $\mathrm{Mg}_{32} \mathrm{O}_{32+x}$ clusters (Fig. 4). The green line at $-7.5 \mathrm{eV}$ is the bulk Fermi level. The overall defect formation energy can be decomposed in two parts: (i) breaking/making the chemical bonds, and (ii) trapping the charge carriers. For the vacancy, the first part amounts to $5.08 \mathrm{eV}$. If we estimate the charging energy as the energy difference between the highest unoccupied molecular orbital and the Fermi level, the vacancy can gain $6.48 \mathrm{eV}$ by trapping two holes (see Fig. 4), resulting in the overall estimated formation energy of $-1.4 \mathrm{eV}$. While for the $\mathrm{O}$-ad-species the estimated energy gain due to hole trapping is much smaller $(2.09 \mathrm{eV})$, the neutral defect has also a much lower formation energy $(0.21 \mathrm{eV})$, resulting in the overall estimated formation energy of $-2.09 \mathrm{eV}$. Thus, the effect of the defect site coordination on the interplay of bond breaking/making and charge-carrier trapping is responsible for the increased stability of O-ad-species.

To confirm the existence of $\mathrm{O}_{1}$ adsorbed species, vibrational-spectroscopy experiments on rugged $\mathrm{MgO}$ surfaces in a controlled $\mathrm{O}_{2}$ atmosphere could look for the vibrational signature of the $\mathrm{O}-\mathrm{O}$ bond stretching of adsorbed $\mathrm{O}_{1}$ with a single trapped hole. We have calculated this frequency using PBE and HSE06 functionals, and the results are 658 and 728 $\mathrm{cm}^{-1}$, respectively. [For reference, PBE (HSE06) frequencies for gas-phase $\mathrm{O}_{2}^{-}$and $\mathrm{O}_{2}^{2-}$ are $1113(1115) \mathrm{cm}^{-1}$ and 645 (669) $\mathrm{cm}^{-1}$, respectively. The experimental value for the gas-phase $\mathrm{O}_{2}^{-}$vibrational frequency is $1108 \mathrm{~cm}^{-1}$ [31], and for $\mathrm{O}_{2}^{2-}$ in various dioxygen-metal complexes it is in the range 790-932 $\mathrm{cm}^{-1}$ [32].]

In conclusion, we find that changing a surface site coordination can change and even reorder the relative stability of surface sites in terms of oxidation or reduction. The applied methodology and the resulting knowledge can be used to design functional materials whose applications rely on surface chemistry.

We are grateful to Matthias Scheffler for support and fruitful discussions. We acknowledge the DFG cluster of excellence "Unifying Concepts in Catalysis" (UniCat) for financial support. S.B. acknowledges the computing facility of HPC, IIT Delhi and Rechenzentrum Garching (RZG) of the Max-Planck Gesellschaft. S.B. acknowledges the financial support from a YSS-SERB research grant, DST, India (Grant No. YSS/2015/001209). S.V.L. is grateful for the support by the Ministry of Education and Science of the Russian Federation in the framework of Increase Competitiveness Program of NUST "MISIS" (No. K2-2016-013) implemented by a governmental decree No. 211 .
[1] Z. Yan, S. Chinta, A. A. Mohamed, J. P. Fackler, and D. W. Goodman, J. Am. Chem. Soc. 127, 1604 (2005).

[2] I. Balint and K.-i. Aika, Appl. Surf. Sci. 173, 296 (2001).

[3] N. A. Richter, S. Sicolo, S. V. Levchenko, J. Sauer, and M. Scheffler, Phys. Rev. Lett. 111, 045502 (2013).

[4] L. Kantorovich and M. Gillan, Surf. Sci. 374, 373 (1997).

[5] D. Vingurt, D. Fuks, M. V. Landau, R. Vidruk, and M. Herskowitz, Phys. Chem. Chem. Phys. 15, 14783 (2013).

[6] R. Richards, W. Li, S. Decker, C. Davidson, O. Koper, V. Zaikovski, A. Volodin, T. Rieker, and K. J. Klabunde, J. Am. Chem. Soc. 122, 4921 (2000).

[7] A. Sternig, O. Diwald, S. Gross, and P. V. Sushko, J. Phys. Chem. C 117, 7727 (2013).

[8] N. Sutradhar, A. Sinhamahapatra, S. K. Pahari, P. Pal, H. C. Bajaj, I. Mukhopadhyay, and A. B. Panda, J. Phys. Chem. C 115, 12308 (2011).

[9] G. Glaspell, Hassan, A. Elzatahry, L. Fuoco, N. R. E. Radwan, and M. S. El-Shall, J. Phys. Chem. B 110, 21387 (2006).

[10] P. V. Sushko, A. L. Shluger, and C. A. Catlow, Surf. Sci. 450, 153 (2000)
[11] S. Sicolo and J. Sauer, J. Phys. Chem. C 117, 8365 (2013).

[12] S. Bhattacharya, S. V. Levchenko, L. M. Ghiringhelli, and M. Scheffler, Phys. Rev. Lett. 111, 135501 (2013).

[13] A semiconducting $p$-doped $\mathrm{MgO}$ can be produced by, e.g., doping with $\mathrm{Li}[28,29]$. Even without intentional doping, nominally pure $\mathrm{MgO}$ becomes a $p$-type semiconductor at temperatures above $800 \mathrm{~K}$ [30], which is comparable to, or even below, the typical temperatures for some of the catalytic applications of $\mathrm{MgO}$.

[14] M. Scheffler and C. Weinert, in Defects in Semiconductors, edited by H. J. v. Bardeleben (Trans. Tech. Publ. Ltd, Switzerland, 1986), pp. 25-30.

[15] X. Yi-Jun, L. Jun-Qian, Z. Yong-Fan, and C. Wen-Kai, Acta Phys.-Chim. Sin. 19, 414 (2003).

[16] N. Bernstein, J. R. Kermode, and G. Csányi, Rep. Prog. Phys. 72, 026501 (2009).

[17] L. Kleinman and D. M. Bylander, Phys. Rev. Lett. 48, 1425 (1982).

[18] V. Blum, R. Gehrke, F. Hanke, P. Havu, V. Havu, X. Ren, K. Reuter, and M. Scheffler, Comput. Phys. Commun. 180, 2175 (2009). 
[19] D. Berger, A. J. Logsdail, H. Oberhofer, M. R. Farrow, C. R. A. Catlow, P. Sherwood, A. A. Sokol, V. Blum, and K. Reuter, J. Chem. Phys. 141, 024105 (2014).

[20] D. Berger, H. Oberhofer, and K. Reuter, Phys. Rev. B 92, 075308 (2015).

[21] See Supplemental Material at http://link.aps.org/supplemental/ 10.1103/PhysRevMaterials.1.071601 for validation of QM/MM methodology, a comparison of the QM/MM embedded model w.r.t periodic calculations, various embedded/unembedded clusters' phase diagrams, convergence of the electrostatic potential w.r.t. the number of point charges, a two-layer phase diagram, various other phase diagrams, and a concentration of defects and error estimation for $\mu_{e}= \pm 0.2 \mathrm{eV}$

[22] J. P. Perdew, K. Burke, and M. Ernzerhof, Phys. Rev. Lett. 77, 3865 (1996).

[23] J. Perdew, K. Burke, and M. Ernzerhof, Phys. Rev. Lett. 78, 1396 (1997).
[24] J. Heyd, G. E. Scuseria, and M. Ernzerhof, J. Chem. Phys. 124, 219906 (2006).

[25] A. Tkatchenko and M. Scheffler, Phys. Rev. Lett. 102, 073005 (2009).

[26] S. Bhattacharya, S. V. Levchenko, L. M. Ghiringhelli, and M. Scheffler, New J. Phys. 16, 123016 (2014).

[27] R. Plass, K. Egan, C. Collazo-Davila, D. Grozea, E. Landree, L. D. Marks, and M. Gajdardziska-Josifovska, Phys. Rev. Lett. 81, 4891 (1998).

[28] Y. Chen, J. Boldu, and V. Orera, Le J. Physique Colloques 41, C6-398 (1980).

[29] M. M. Tardío, R. Ramírez, R. González, and Y. Chen, Phys. Rev. B 66, 134202 (2002).

[30] F. Freund, M. M. Freund, and F. Batllo, J. Geophys. Res.: Solid Earth 98, 22209 (1993).

[31] K. M. Ervin, I. Anusiewicz, P. Skurski, J. Simons, and W. C. Lineberger, J. Phys. Chem. A 107, 8521 (2003).

[32] L. Vaska, Acc. Chem. Res. 9, 175 (1976). 